\title{
Stock Market Integration in China: Evidence from the Asymmetric DCC Model and Copula Approach
}

\author{
Xiao Jing Cai ${ }^{1}$, Shuairu Tian ${ }^{1}$, Shigeyuki Hamori ${ }^{2}$ \\ ${ }^{1}$ Graduate School of Economics, Kobe University, 2-1, Rokkodai, Nada-Ku, Kobe 657-8501, Japan \\ ${ }^{2}$ Faculty of Economics, Kobe University, 2-1, Rokkodai, Nada-Ku, Kobe 657-8501, Japan \\ Correspondence: Shigeyuki Hamori, Faculty of Economics, Kobe University, 2-1, Rokkodai, Nada-Ku, Kobe 657-8501, \\ Japan.
}

Received: October 24, 2016

doi:10.11114/aef.v4i2.2010
Accepted: November 16, $2016 \quad$ Available online: November 24, 2016

URL: http://dx.doi.org/10.11114/aef.v4i2.2010

\begin{abstract}
We investigate the dynamic dependence structure between the daily stock returns of the A and B shares of the Shanghai and Shenzhen stock markets in China, using time-varying conditional copula and asymmetric dynamic conditional correlation models. We find that the Shanghai market's A and B shares are more integrated than those of the Shenzhen market. Further, the dynamic dependences between the shares for both markets are asymmetric and lower-tailed, and an increasing correlation with the opening up of the B shares market to Chinese citizens around 2001 is evident.
\end{abstract}

Keywords: asymmetric dynamic conditional correlation, time-varying copula, dynamic dependence, Chinese stock markets

\section{Introduction}

Chinese stock markets have developed rapidly in the past two decades, leading to increased scholarly attention (Xu 2000; Jin 2015; Luo et al. 2015; Sun and Tong 2000). According to data released by the Shanghai and Shenzhen Stock Exchanges (SEs), the total equity market capitalization of the A and B shares of their respective markets was CNY 19.6 trillion (about USD 3.09 trillion) in April 2013. A (B) shares listed on the Shanghai and Shenzhen SEs are named Shanghai A (B) shares and Shenzhen A (B) shares. China is unique in that trading in its equity markets is completely segmented between A shares and B shares for Chinese and foreign investors, respectively. A shares (including Shanghai A shares and Shenzhen A shares) are traded by domestic citizens in Renminbi (RMB), while B shares (including Shanghai B shares and Shenzhen B shares) are denominated in US dollars on the Shanghai SE and in HK dollars on the Shenzhen SE. The main reason behind this segmentation is that the Chinese government wants to attract foreign capital without worrying about the loss of ownership rights.

Although the Chinese government has permitted the same company to issue both A and B shares, compared with the rather large A shares market, which was worth CNY 18.71 trillion in April 2013, the B shares market is much smaller (only CNY 0.17 trillion in 2013) and thus less active as an investment avenue. Bailey (1994) first documented that the selling prices of B shares are discounted relative to their A shares counterparts and suggested that the lower cost of capital for Chinese citizens may explain the low number of investment alternatives for local investors. Indeed, in 2001, the Chinese Securities Regulatory Commission announced a new policy that allowed Chinese citizens to trade in B shares. This triggers two questions: (1) Are the prices of A and B shares correlated? (2) Should B shares be merged into the A shares market? While recent research has highlighted China's increasing integration into international markets (Wang et al. 2011; Li 2013; Chien et al. 2015; Zhu et al. 2015), few studies investigate market segmentation and integration in China. Hence, it is necessary to examine the dependence structure between and integration of the A and B shares markets in China to guide both investment and policymaking.

In this study, we aim to capture the joint behavior of A and B shares by using two approaches. The first method considered here is the asymmetric dynamic conditional correlation (ADCC) model proposed by Cappiello et al. (2006), which can capture conditional asymmetric effects in correlation dynamics. This approach has been applied to commodities (Toyoshima et al. 2013), stocks (Toyoshima and Hamori 2013), bonds (Toyoshima et al. 2012), and bank credit default swap markets (Tamakoshi and Hamori 2013). 
The second method we consider is the copula approach. Copula theory, developed by Sklar (1959), explains that a joint distribution can be decomposed into its marginal distributions and a copula, which can characterize the dependence between variables. The concept of the conditional copula was introduced by Patton (2006b), who adapted the copula framework to a time-series case by allowing for time variation in the parameters of marginal distributions. The advantage of the copula approach is that it allows for different types of dependences (e.g., symmetric, asymmetric, lower/upper tail dependence).

Our contributions to the literature can be summarized as follows. We first compensate for the dearth of studies of the segmentation and integration of Chinese stock markets. We find that both the level and the variability of the joint dependence of the A and B shares of the Shanghai SE are larger than those of the Shenzhen SE, indicating that A shares are more integrated with B shares in the former. We also show that the dynamic rotated-Gumbel copula functions fit situations of dependence better than the time-varying Student's t-copula functions for both markets according to the Cramer-von Mises (CvM) goodness-of-fit test (see the Appendix for more details), which suggests that the dynamic conditional dependences for both markets are asymmetric and more likely to be lower tail-dependent.

The remainder of the article is organized as follows. Section 2 introduces the model for the marginal distribution, ADCC model, and time-varying conditional copula models. Section 3 describes our data. In Section 4, we discuss the results of our estimation. Section 5 concludes.

\section{Model Specifications}

\subsection{Model for the Marginal Distributions}

The estimation procedure in copula modelling begins with the identification of the marginal distributions and estimation of their parameters. We fit univariate GARCH models to each return series. Different GARCH-type models and varying lag lengths are possible for different return series, and the best model is typically selected by using the Akaike information criterion (AIC) and Bayesian information criterion (BIC).

If we consider $y_{t}$ for the $t=1, \ldots, T$ asset returns series, all the AR (1)-GARCH specifications can be given by the following characteristics:

$$
\begin{aligned}
y_{i, t} & =\phi_{0, i}+\phi_{1, i} y_{i, t-1}+\varepsilon_{i, t}, i=1, \ldots, n \\
\sigma_{i, t}^{\delta} & =\omega_{i}+\sum_{q=1}^{Q} \beta_{i, q} \sigma_{i, t-q}^{\delta}+\sum_{p=1}^{P} \alpha_{i, p}\left|\varepsilon_{i, t-p}\right|^{\delta}+\sum_{\iota=1}^{J} \gamma_{i, l}\left|\varepsilon_{i, t-\iota}\right|^{\delta} s_{i, t-\iota}
\end{aligned}
$$

where $s_{i, t}$ is an indicator function that takes the value of one if $\varepsilon_{i, t}$ is negative and zero otherwise. As Table 1 shows, the GARCH and Glosten-Jagannathan-Runkle (GJR)-GARCH models parameterize the conditional variance, whereas the absolute value (AV) GARCH and threshold GARCH (TGARCH) models directly parameterize the conditional standard deviation. In addition, the GJR-GARCH and TGARCH specifications introduce asymmetries in the variance and standard deviation equations, respectively.

Table 1. GARCH Specifications

$\begin{array}{llll}\text { GARCH } & \text { Bollerslev (1986) } & \delta=2 & \iota=0 \\ \text { GJR-GARCH } & \text { Glosten et al. (1993) } & \delta=2 & \iota \neq 0 \\ \text { AVGARCH } & \text { Taylor (1986) } & \delta=1 & \iota=0 \\ \text { TGARCH } & \text { Zakoian (1994) } & \delta=1 & \iota \neq 0\end{array}$

We assume the error term $\varepsilon_{i, t}$ follows Hansen's (1994) skewed Student's t-distribution as follows:

$$
d(z \mid \eta, \lambda)=\left\{\begin{array}{l}
b c\left(1+\frac{1}{\eta-2}\left(\frac{b z+a}{1-\lambda}\right)^{2}\right)^{-\frac{\eta+1}{2}} \text { if } z<-\frac{a}{b} \\
b c\left(1+\frac{1}{\eta-2}\left(\frac{b z+a}{1+\lambda}\right)^{2}\right)^{-\frac{\eta+1}{2}} \text { if } z \geq-\frac{a}{b}
\end{array}\right.
$$

where $a \equiv 4 \lambda c \frac{\eta-2}{\eta-1}, b^{2} \equiv 1+3 \lambda-a^{2}$, and $c \equiv \frac{\Gamma\left(\frac{\eta+1}{2}\right)}{\sqrt{\pi(\eta-2) \Gamma\left(\frac{\eta}{2}\right)}} . \lambda$ and $\eta$ are the asymmetry skewness parameter and degree of freedom parameter, respectively.

If the random variable $z$ has the density $d(z \mid \eta, \lambda)$, we write $z \sim S T(z \mid \eta, \lambda)$. An inspection of the various formulas reveals that this density is defined for $2<\eta<\infty$ and $-1<\lambda<1$. If $\lambda=0$, Hansen's skewed Student's t-distribution is then reduced to the traditional Student's t-distribution, which is not skewed. If, in addition, $\eta \rightarrow \infty$, the Student's t-distribution collapses to the normal density. 


\subsection{ADCC Model}

Here, we briefly review the ADCC model. Specifically, based on the conditional volatilities from equation (2), we calculate the conditional correlations from the conditional covariance matrix as

$$
H_{t}=D_{t} R_{t} D_{t}
$$

where $H_{t}$ is an $N \times N$ positive definite matrix such that $H_{t}$ is the conditional variance matrix of $y_{t}$. $D_{t}$ is an $N \times N$ diagonal matrix of the standard deviations of residual returns:

$$
R_{t}=\operatorname{diag}\left(Q_{t}\right)^{-1 / 2} Q_{t} \operatorname{diag}\left(Q_{t}\right)^{-1 / 2}
$$

where $R_{t}$ is the correlation matrix constituted by the correlation $\rho_{i j, t}$. To parameterize the correlation coefficient $\rho_{t}$, we assume that $Q_{t}$ is autoregressive:

$$
Q_{t}=\left(\bar{Q}-A^{\prime} \bar{Q} A-B^{\prime} \bar{Q} B-G^{\prime} \bar{N} G\right)+A^{\prime} z_{t-1} z_{t-1}^{\prime} \mathrm{A}+B^{\prime} Q_{t-1} B+G^{\prime} n_{t-1} n_{t-1}^{\prime} \mathrm{G}
$$

where $\bar{Q}$ is the $N \times N$ unconditional correlation coefficient matrix, $\bar{N}$ is the unconditional covariance matrix of $n_{t}$, $z_{t-1} z^{\prime}{ }_{t-1}$ is the lagged function of the standardized residuals derived from the univariate GARCH estimation, and $n_{t}$ is a function indicator that takes the value of one if the residuals are negative and zero otherwise. $\mathrm{A}, \mathrm{B}$, and $\mathrm{G}$ are diagonal matrices.

The scalar ADCC can be specified as

$$
Q_{t}=(\bar{Q}-a \bar{Q}-b \bar{Q}-g \bar{N})+a z_{t-1} z_{t-1}^{\prime}+b Q_{t-1}+g n_{t-1} n_{t-1}^{\prime}
$$

where the restriction conditions are $a, b \geq 0$ and $a+b+\tau g<1$, and $\tau$ is the maximum eigenvalue of $Q_{t}{ }^{-1 / 2} \bar{N} Q_{t}{ }^{-1 / 2}$. For this model, the parameter $b$ represents the degree of inertia in time-varying conditional correlations, whereas the parameter $a$ represents the degree of perturbation to $\rho_{i j, t}$ and the parameter $g$ introduces asymmetric effects into the model.

\subsection{Copula Distribution Functions}

In accordance with Patton (2006b), we consider the information set $\mathcal{F}_{t-1}$. If we assume that $X=\left(x_{1}, x_{2}\right)^{\prime}$ is a random vector with a two-dimensional conditional distribution function $\left(\cdot \mid \mathcal{F}_{t-1}\right)$, and that its conditional marginal distributions are $F_{1}\left(\cdot \mid \mathcal{F}_{t-1}\right), F_{2}\left(\cdot \mid \mathcal{F}_{t-1}\right)$, then there exists a conditional copula $C$ such that for all $\mathrm{X} \in \mathbb{R}^{2}$,

$$
F\left(x_{1}, x_{2} \mid \mathcal{F}_{t-1}\right)=C\left(F_{1}\left(x_{1} \mid \mathcal{F}_{t-1}\right), F_{2}\left(x_{2} \mid \mathcal{F}_{t-1}\right) \mid \mathcal{F}_{t-1}\right)
$$

The conditional copula $C$ is uniquely determined in (8) if the conditional marginal distributions $F_{1}\left(\cdot \mid \mathcal{F}_{t-1}\right)$ and $F_{2}\left(\cdot \mid \mathcal{F}_{t-1}\right)$ are continuous. Then, for any $u_{i}$ in $[0,1]^{2}$ :

$$
C\left(u_{1}, u_{2} \mid \mathcal{F}_{t-1}\right)=F\left(F_{1}^{-1}\left(u_{1}\right), F_{2}^{-1}\left(u_{2}\right) \mid \mathcal{F}_{t-1}\right)
$$

where $F_{i}^{-1}\left(\cdot \mid \mathcal{F}_{t-1}\right)$ is the generalized inverse of $F_{i}\left(\cdot \mid \mathcal{F}_{t-1}\right)$.

Conditional upper tail dependence $\lambda^{U}$ and conditional lower tail dependence $\lambda^{L}$ are defined as follows:

$$
\begin{aligned}
& \lambda^{U}=\lim _{u \rightarrow 1} \operatorname{Pr}\left[x_{2}>F_{2}^{-1}(u) \mid x_{1}>F_{1}^{-1}(u), \mathcal{F}_{t-1}\right]=\lim _{u \rightarrow 1} \frac{\left[1-2 u+C\left(u, u \mid \mathcal{F}_{t-1}\right)\right]}{1-u} \\
& \lambda^{L}=\lim _{u \rightarrow 0} \operatorname{Pr}\left[x_{2} \leq F_{2}^{-1}(u) \mid x_{1} \leq F_{1}^{-1}(u), \mathcal{F}_{t-1}\right]=\lim _{u \rightarrow 0} \frac{C\left(u, u \mid \mathcal{F}_{t-1}\right)}{u}
\end{aligned}
$$

If $\lambda^{U} \in(0,1]$, the random variables $x_{1}$ and $x_{2}$ are asymptotically dependent in the conditional upper tail. On the contrary, if $\lambda_{u}=0$, they are asymptotically independent in the conditional upper tail. Conditional lower tail dependence can be described in a similar way. If $\lambda_{L} \in(0,1]$, then $C$ has conditional lower tail dependence. If $\lambda_{L}=0$, $C$ has conditional lower tail independence.

The details provided here concern the copula functions for two widely used copula families: the elliptical copula family and Archimedean copula family. In this article, we select the time-varying Student's t-copula to calculate the symmetric dynamic conditional dependence for the elliptical copula. For the Archimedean copula, we employ the rotated-Gumbel copula to investigate the asymmetric dynamic conditional dependence, particularly as the rotated-Gumbel copula can measure extreme co-movements in the conditional lower tail.

[1] The Student's t-copula: $\theta=(v, \rho)$.

The cumulative distribution function (CDF) of the Student's t-copula introduced by Dias and Embrechts (2004) and Patton (2006a) is as follows: 


$$
C_{v, \rho}^{t}\left(u_{1}, u_{2}\right)=T_{2, v, \rho}\left(t_{v}^{-1}\left(u_{1}\right), t_{v}^{-1}\left(u_{2}\right)\right)
$$

and the density function of the Student's t-copula is

$$
c_{v, \rho}^{t}\left(u_{1}, u_{2}\right)=\frac{1}{\sqrt{1-\rho^{2}}} \frac{\Gamma\left(\frac{v+2}{2}\right) \Gamma\left(\frac{v}{2}\right)\left(1+\frac{1}{v} \psi^{\prime} \Omega^{-1} \psi\right)^{-\frac{v+2}{2}}}{\left(\Gamma\left(\frac{v+1}{2}\right)\right)^{2} \prod_{i=1}^{2}\left(1+\frac{1}{v} \psi_{i}^{2}\right)^{-\frac{v+1}{2}}}
$$

where $\psi=\left(t_{v}^{-1}\left(u_{1}\right), t_{v}^{-1}\left(u_{2}\right)\right)^{\prime}$ and $\Omega$ is the $2 \times 2$ correlation matrix with $\rho$ as the correlation between $u_{1}$ and $u_{2}$. $T_{2, v, \rho}$ is the Student's t-CDF, with $v$ degrees of freedom and correlation $\rho$. The time-varying copula methodology we adopt is that of Patton (2006b), who allows for time variation in the conditional copula by assuming that the dependence parameter $\left(\rho_{t}\right)$ evolves over time according to an equation that follows an autoregressive moving average or ARMA (1, 10) process, with an autoregressive component for capturing persistence in the dependence parameter and a forcing variable for capturing variation in dependence. For instance, the time-varying Student's t-copula evolution equations are given by

$$
\rho_{t}=\Lambda\left\{\omega+\beta \rho_{t-1}+\alpha \frac{1}{10} \sum_{j=1}^{10} T_{v}^{-1}\left(u_{1, t-j}\right) T_{v}^{-1}\left(u_{2, t-j}\right)\right\}
$$

where $\Lambda$ is a logistic transformation used to keep the parameter $\rho$ in its $(-1,1)$ interval of the copula function at all times, and $\Lambda(x) \equiv\left(1-e^{-x}\right)\left(1+e^{-x}\right)^{-1}=\tanh \left(\frac{x}{2}\right)$. The parameter $\alpha$ denotes the adjustment in the dependence process, while $\beta$ represents the degree of persistence.

The Student's t-copula has symmetrical tail dependence, and the upper tail dependency is $\lambda^{U}=2-2 t_{v+1}(\sqrt{v+1} \sqrt{1-\rho} / \sqrt{1+\rho})$. Because of the symmetry property, the lower tail dependency $\lambda^{L}$ can be easily obtained.

[2] The rotated-Gumbel copula: $(\theta=\delta, \delta \geq 1)$. We first introduce the Gumbel copula first discussed by Gumbel (1960) and expanded upon by Nelsen (1999). It takes the form:

$$
\begin{aligned}
& C_{\delta}^{G}\left(u_{1}, u_{2}\right)=\exp \left(-\left[\left(-\ln u_{1}\right)^{\delta}+\left(-\ln u_{2}\right)^{\delta}\right]^{\frac{1}{\delta}}\right) \\
& c_{\delta}^{G}\left(u_{1}, u_{2}\right)=\frac{C_{\delta}^{G}\left(u_{1}, u_{2} ; \delta\right)\left(\ln u_{1} \ln u_{2}\right)^{\delta-1}\left\{\left[\left(-\ln u_{1}\right)^{\delta}+\left(-\ln u_{2}\right)^{\delta}\right]^{\frac{1}{\delta}}+\delta-1\right\}}{u_{1} u_{2}\left[\left(-\ln u_{1}\right)^{\delta}+\left(-\ln u_{2}\right)^{\delta}\right]^{2-\frac{1}{\delta}}}
\end{aligned}
$$

where $C_{\delta}^{G}$ and $c_{\delta}^{G}$ are the CDF and density functions of the Gumbel copula, respectively.

The dependency structures of the Gumbel copulas are asymmetric: $\lambda^{U}=2-2^{1 / a}$ and $\lambda^{L}=0$.

The rotated-Gumbel copula $(\theta=\delta, \delta \geq 1)$ has the following form:

$$
C_{\delta}^{R G}\left(u_{1}, u_{2} \mid \delta\right)=u_{1}+u_{2}-1+C_{\delta}^{G}\left(1-u_{1}, 1-u_{2} \mid \delta\right)
$$

where $C_{\delta}^{G}$ corresponds to the Gumbel copula. The dependence parameter $\delta$ follows the process:

$$
\delta_{t}=\Lambda\left\{\omega+\beta \delta_{t-1}+\alpha \frac{1}{10} \sum_{j=1}^{10}\left|u_{1, t-j}-u_{2, t-j}\right|\right\}
$$

where $\Lambda(x)=1+x^{2}$ is a polynomial transformation to ensure that $\delta_{t} \in[1, \infty)$. The parameter $\alpha$ denotes the variability of the dependence and the parameter $\beta$ denotes the persistence effect. The rotated-Gumbel copula has only lower tail dependence $\left(\lambda^{U}=0\right)$, given by $\lambda^{L}=2-2^{1 / \delta}$.

\subsection{Estimation}

The ADCC model is estimated by using the quasi-maximum likelihood method. Following Engle (2002), the likelihood can be split into two parts: the term of volatility $\left(l_{\gamma}(\theta)\right)$ and the term of correlation $\left(l_{c}(\theta, \varnothing)\right)$. Then, the two terms can be maximized as follows:

$$
\widehat{\theta}=\operatorname{argmax}\left\{l_{\gamma}(\theta)\right\}
$$

The parameters of the copulas are estimated in two steps. The log-likelihood function for $\left\{x_{t}\right\}_{t=1}^{T}$ is

$$
\begin{aligned}
\mathcal{L}(\boldsymbol{\theta}) & =\sum_{t=1}^{T} \ln f\left(x_{1 t}, x_{2 t} ; \boldsymbol{\theta}\right) \\
& =\sum_{t=1}^{T} \ln f_{1}\left(x_{1 t} ; \theta_{1}\right)+\sum_{t=1}^{T} \ln f_{2}\left(x_{2 t} ; \theta_{2}\right)+\sum_{t=1}^{T} \ln c\left(F_{1}\left(x_{1 t} ; \theta_{1}\right), F_{2}\left(x_{2 t} ; \theta_{2}\right) ; \theta_{3}\right)
\end{aligned}
$$

where $T$ is the number of observations and $\boldsymbol{\theta}=\left(\theta_{1}^{\prime}, \theta_{2}^{\prime}, \theta_{3}{ }^{\prime}\right)^{\prime}$ are the parameters in the conditional marginal densities $f_{1}\left(. \mid \mathcal{F}_{t-1}\right)$ and $f_{2}\left(. \mid \mathcal{F}_{t-1}\right)$, as well as the copula shape parameters. The log-likelihood is decomposed into two parts, 
with the first two terms related to the marginal estimation and the last term related to the copula.

\section{Data}

The data set comprises the daily closing price indices of the A and B shares markets of the Shanghai and Shenzhen SEs from December 31, 1999 to April 22, 2013, amounting to 3472 observations. All data were obtained from DataStream. Table 2 presents the descriptive statistics and several basic statistical tests performed on the index returns. The sample period runs from January 3, 2000 to April 22, 2013. As shown in Table 2, all markets display excess kurtosis. In addition, the returns show negative skewness, except for the Shenzhen SE's B shares. The Jarque-Bera test shows that the null hypothesis of normality is rejected in all cases. Therefore, it is appropriate to use the skewed Student's $\mathrm{t}$-distribution to deal with these properties.

Table 2. Descriptive Statistics on Daily Stock Returns

\begin{tabular}{lllll}
\hline & Shanghai A & Shanghai B & Shenzhen A & Shenzhen B \\
\hline Mean & $0.0601 \mathrm{E}-03$ & $0.2427 \mathrm{E}-03$ & $0.1038 \mathrm{E}-03$ & $0.2856 \mathrm{E}-03$ \\
SD & 0.0068 & 0.0090 & 0.0074 & 0.0084 \\
Skewness & -0.0817 & -0.0113 & -0.3579 & 0.0729 \\
Kurtosis & 7.6952 & 8.0229 & 6.5782 & 7.6520 \\
Jarque-Bera & $3192.174 * * *$ & $3648.851^{* * *}$ & $1925.784 * * *$ & $3132.911^{* * *}$
\end{tabular}

Notes: SD stands for standard deviation; *** represents significance at the $1 \%$ level; Jarque-Bera corresponds to the Jarque-Bera test statistics.

Table 3 presents the Pearson, Spearman, and Kendall correlations. The Pearson correlations show linear associations, while the Spearman and Kendall correlations are nonparametric rank correlations. As illustrated in Table 3, the Shanghai SE's A and B shares have a higher correlation than those of the Shenzhen SE.

Table 3. Correlation Estimates between the A and B Shares

\begin{tabular}{llll}
\hline & Pearson & Spearman & Kendall \\
\hline Shanghai A-B & 0.734 & 0.768 & 0.597 \\
Shenzhen A-B & 0.729 & 0.749 & 0.570 \\
\hline
\end{tabular}

Notes: All values are significant at the $1 \%$ level.

\section{Estimation Results}

First, we employ a univariate AR-GARCH process to estimate the marginal distributions of the A and B shares in both SEs. Table 4 presents our marginal distribution results. According to the AIC and BIC, we select a GJR-GARCH $(1,1,1)$ model for the Shanghai SE A shares, Shanghai SE B shares, and Shenzhen SE B shares, and a $\operatorname{GARCH}(1,1)$ model for the Shenzhen SE A shares. The conditional variance term coefficients $(\beta)$ of the four stock indices are all statistically significant at the $1 \%$ level and close to 1, implying a high level of persistence. The estimates of the asymmetric parameters $\gamma$ are statistically significant at the $1 \%$ level and positive. Both markets exhibit the leverage effect, whereby negative shocks have a greater impact on future volatility levels than positive shocks of the same magnitude. Furthermore, the entire skew term coefficient $\lambda$ is statistically and positively significant at the $1 \%$ level, which indicates that the skewed Student's t-distribution fits the models well. The degree of freedom parameters $(\eta)$ are statistically significant at the $1 \%$ level for values exceeding 2 , suggesting that the tails of the error terms are heavier compared with the normal distribution. Moreover, the statistics and p-values of both $Q^{2}(10)$ at lag 10 and $Q^{2}(20)$ at lag 20 indicate that the null hypothesis of no autocorrelation up to orders 10 and 20 for standardized squared residuals cannot be rejected, which supports the adoption of our marginal specification. 
Table 4. Estimation Results of the Marginal Distribution

\begin{tabular}{|c|c|c|c|c|}
\hline & Shanghai A & Shanghai B & Shenzhen A & Shenzhen B \\
\hline \multicolumn{5}{|l|}{ Mean model } \\
\hline \multirow[t]{2}{*}{$\phi_{0}$} & $8.430 \mathrm{E}-06$ & $1.238 \mathrm{E}-04$ & $3.552 \mathrm{E}-05$ & $2.010 \mathrm{E}-04 *$ \\
\hline & (9.119E-05) & $(0.0001)$ & (9.754E-05) & $(1.270 \mathrm{E}-04)$ \\
\hline \multirow[t]{2}{*}{$\phi_{1}$} & 0.002 & $0.080 * * *$ & $0.112 * * *$ & $0.082 * * *$ \\
\hline & $(0.016)$ & $(0.019)$ & $(0.017)$ & $(0.017)$ \\
\hline \multicolumn{5}{|l|}{ Variance model } \\
\hline \multirow[t]{2}{*}{$\omega$} & $5.201 \mathrm{E}-07 * * *$ & $4.60 \mathrm{E}-06 * * *$ & $7.093 \mathrm{E}-07 * * *$ & $6.222 \mathrm{E}-06^{* * *}$ \\
\hline & $(2.232 \mathrm{E}-07)$ & $(4.519 \mathrm{E}-07)$ & $(2.629 \mathrm{E}-07)$ & $(6.694 \mathrm{E}-07)$ \\
\hline \multirow[t]{2}{*}{$\alpha$} & $0.041 * * *$ & $0.116^{* * *}$ & $0.059 * * *$ & $0.106 * * *$ \\
\hline & $(0.004)$ & $(0.008)$ & $(0.003)$ & $(0.009)$ \\
\hline \multirow[t]{2}{*}{$\beta$} & $0.931 * * *$ & $0.802 * * *$ & $0.926 * * *$ & $0.784 * * *$ \\
\hline & $(0.003)$ & $(0.008)$ & $(0.003)$ & $(0.012)$ \\
\hline \multirow[t]{2}{*}{$\gamma$} & $0.032 * * *$ & $0.056 * * *$ & - & $0.033 * * *$ \\
\hline & $(0.006)$ & $(0.011)$ & & $(0.012)$ \\
\hline \multirow[t]{2}{*}{$\lambda$} & $0.955 * * *$ & $0.97 * * *$ & $0.893 * * *$ & $0.962 * * *$ \\
\hline & $(0.017)$ & $(0.022)$ & $(0.019)$ & $(0.019)$ \\
\hline \multirow[t]{2}{*}{$\eta$} & $3.054 * * *$ & $2.412 * * *$ & $4.536 * * *$ & $3.008 * * *$ \\
\hline & $(0.285)$ & $(0.142)$ & $(0.378)$ & $(0.157)$ \\
\hline \multirow[t]{2}{*}{$Q^{2}(10)$} & 2.853 & 9.036 & 3.141 & 4.849 \\
\hline & [0.985] & [0.529] & {$[0.978]$} & {$[0.901]$} \\
\hline \multirow[t]{2}{*}{$Q^{2}(20)$} & 8.364 & 16.714 & 7.691 & 9.32 \\
\hline & [0.989] & {$[0.671]$} & [0.994] & [0.979] \\
\hline
\end{tabular}

Notes: Standard errors are in parentheses. The numbers in square brackets are p-values. *, **, and *** represent significance at the $10 \%, 5 \%$, and $1 \%$ levels, respectively. The $\mathrm{Q}^{2}(s)$ statistics are the standardized squared residual statistics of the Ljung-Box test for the null hypothesis that there is no autocorrelation up to order $s$.

We then estimate the asymmetric dynamic conditional correlations by employing our ADCC models and calculate the dynamic conditional dependences by using the time-varying copula functions. For comparison with the time-varying rotated-Gumbel copula function, we also estimate the parameters of the time-varying Student's t-copula models. The results are reported in Table 5.

Table 5. Estimation Results for the ADCC Model and Copula Functions

\begin{tabular}{|c|c|c|}
\hline & Shanghai A-B & Shenzhen A-B \\
\hline \multicolumn{3}{|l|}{ ADCC } \\
\hline \multirow[t]{2}{*}{$a$} & $0.027 * * *$ & $0.019 * *$ \\
\hline & $(0.010)$ & $(0.006)$ \\
\hline \multirow[t]{2}{*}{$b$} & $0.952 * * *$ & $0.959 * * *$ \\
\hline & $(0.006)$ & $(0.010)$ \\
\hline \multirow[t]{2}{*}{$g$} & $0.035 * *$ & $0.021 * *$ \\
\hline & $(0.01)$ & $(0.009)$ \\
\hline \multicolumn{3}{|c|}{ Time-varying Student's t-copula } \\
\hline \multirow[t]{2}{*}{$\omega$} & $0.246^{* * *}$ & $0.155^{* * *}$ \\
\hline & $(0.056)$ & $(0.0001)$ \\
\hline \multirow[t]{2}{*}{$\alpha$} & $0.193 * * *$ & $0.157 * * *$ \\
\hline & $(0.020)$ & $(0.016)$ \\
\hline \multirow[t]{2}{*}{$\beta$} & $0.934 * * *$ & $0.883 * * *$ \\
\hline & $(0.0001)$ & $(0.026)$ \\
\hline \multirow[t]{2}{*}{$v$} & $0.198 * * *$ & $0.182 * * *$ \\
\hline & $(0.023)$ & $(0.022)$ \\
\hline \multirow[t]{2}{*}{ CvM statistics } & $91.33 * * *$ & $92.783 * * *$ \\
\hline & {$[0.00]$} & {$[0.00]$} \\
\hline \multicolumn{3}{|c|}{ Time-varying rotated-Gumbel copula } \\
\hline \multirow[t]{2}{*}{$\omega$} & $0.039 * * *$ & 0.028 \\
\hline & $(0.004)$ & $(0.026)$ \\
\hline \multirow[t]{2}{*}{$\alpha$} & $0.201 * * *$ & $0.181 * * *$ \\
\hline & $(0.027)$ & $(0.050)$ \\
\hline \multirow[t]{2}{*}{$\beta$} & $0.908 * * *$ & $0.8 * * *$ \\
\hline & $(0.063)$ & $(0.025)$ \\
\hline \multirow[t]{2}{*}{ CvM statistics } & 1.134 & 0.587 \\
\hline & {$[0.11]$} & {$[0.58]$} \\
\hline
\end{tabular}


For the ADCC model, the innovation parameters $a$ for the Shanghai and Shenzhen SEs are 0.019 and 0.027, respectively, and the corresponding smoothness parameters $b$ are 0.952 and 0.959 . They are all statistically significant at the $1 \%$ level, indicating a high level of persistence for both markets. The asymmetric terms $g$ are statistically significant at the 5\% level for both SEs, which indicates that negative shocks have a greater impact on dynamic correlation levels than positive shocks in both cases. For both the copula functions, the parameters $\beta$ represent the degree of persistence, and $\alpha$ captures the adjustment in the dependence process. The parameters $\beta$ of both time-varying copula functions are statistically significant at the $1 \%$ level, denoting a high degree of dependence persistence for both markets.

Significant variations over time in the dependences between the A and B shares for both markets are also present, as indicated by the values of $\alpha$. The A and B shares for the Shanghai SE have greater dependence than those of the Shenzhen SE, as indicated by their dependence levels described by $\omega$, which concur with the results in Table 3 . Thus, the results demonstrate that the Shanghai SE has not only higher levels of conditional variability but also greater dependence than the Shenzhen SE. This outcome provides useful insights into how to practice active risk portfolio management in Chinese stock markets.

We also present the statistics and p-values from the CvM goodness-of-fit test under the null hypothesis that the empirical distributions fit the theoretical cumulative distribution correctly (see the Appendix). According to the CvM goodness-of-fit test, the null hypothesis for the time-varying Student's t-copula is rejected at the 1\% level for both SEs, indicating the time-varying Student's t-copulas do not fit the dynamic dependence between the A and B shares well. On the contrary, the time-varying rotated-Gumbel copulas fit the model well for both SEs. Thus, we select the time-varying rotated-Gumbel copula functions to model the conditional dynamic dependence for the two markets, demonstrating evidence of asymmetric and tail dependence for both markets. Tail dependences are useful for examining the joint extreme events affecting financial returns during periods of high volatility or a market crash (Hu 2010).

Figure 1 illustrates the time paths of the conditional lower tail dependences for the rotated-Gumbel copula. We find the lower tail dependence between the A and B shares for both markets increases with great volatility around 2001, which suggests that the A and B shares for both markets are more likely to react to the same negative extreme shocks after the opening up of the B shares market to Chinese citizens in 2001. Moreover, we find that the Shanghai SE has a higher degree of lower tail dependence than the Shenzhen SE, which is consistent with the results reported in Table 5.
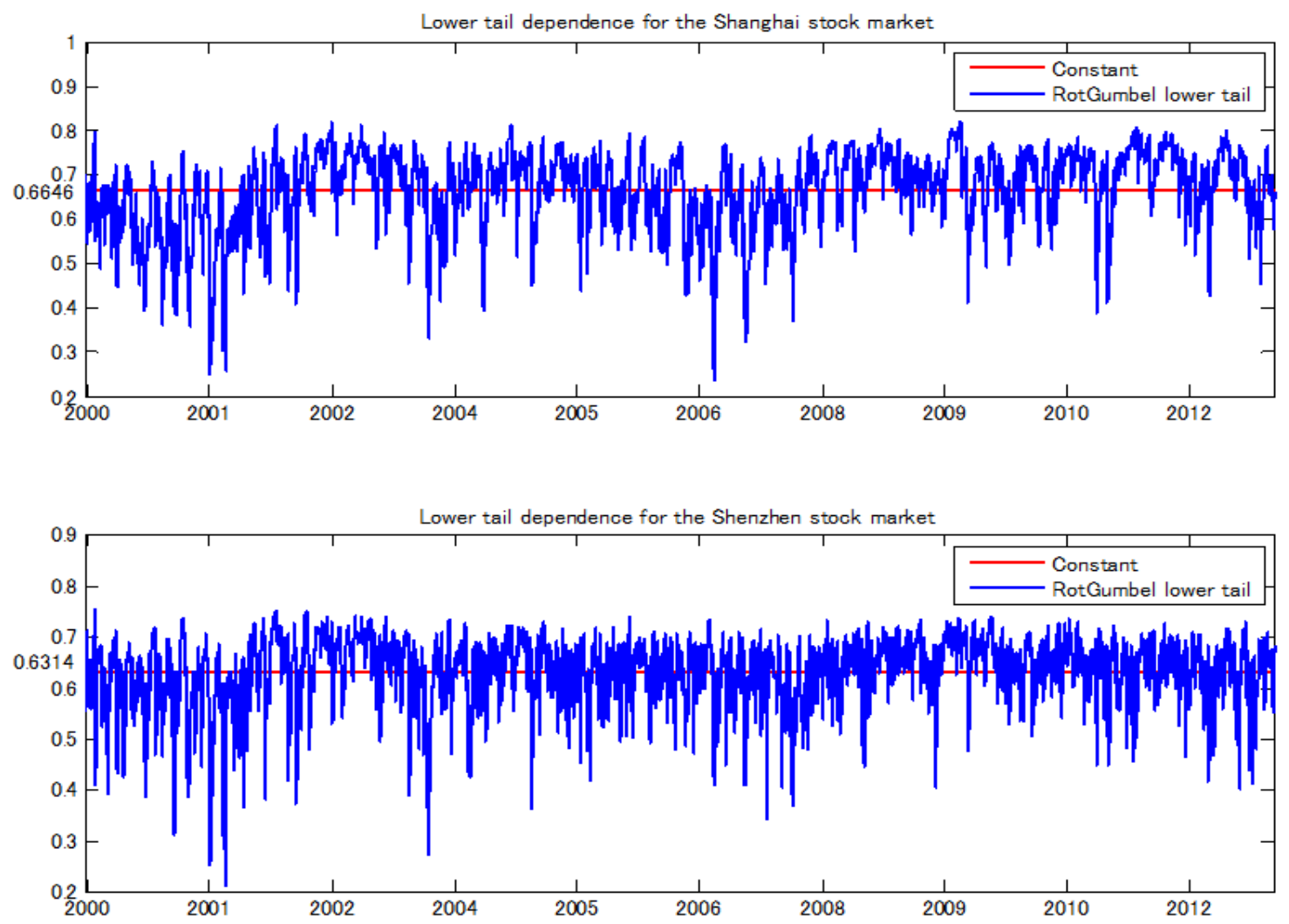

Figure 1. Time-Varying Conditional Lower Tail Dependence for the Shanghai SE (a) and Shenzhen SE (b)

Figure 2 shows the dynamics of the conditional correlations and time-varying parameters of the rotated-Gumbel copula. Since the opening up of the B shares market to Chinese citizens, the A-B shares return correlations have risen for both 
SEs. Moreover, there have been signs of increasing correlations between the A and B shares for both markets since the 2007-2008 global financial crisis. This finding demonstrates that when the market is down, risk diversification is less effective because of the greater dependence. Further, the A shares are more integrated with the B shares in the Shanghai SE than in the Shenzhen SE.
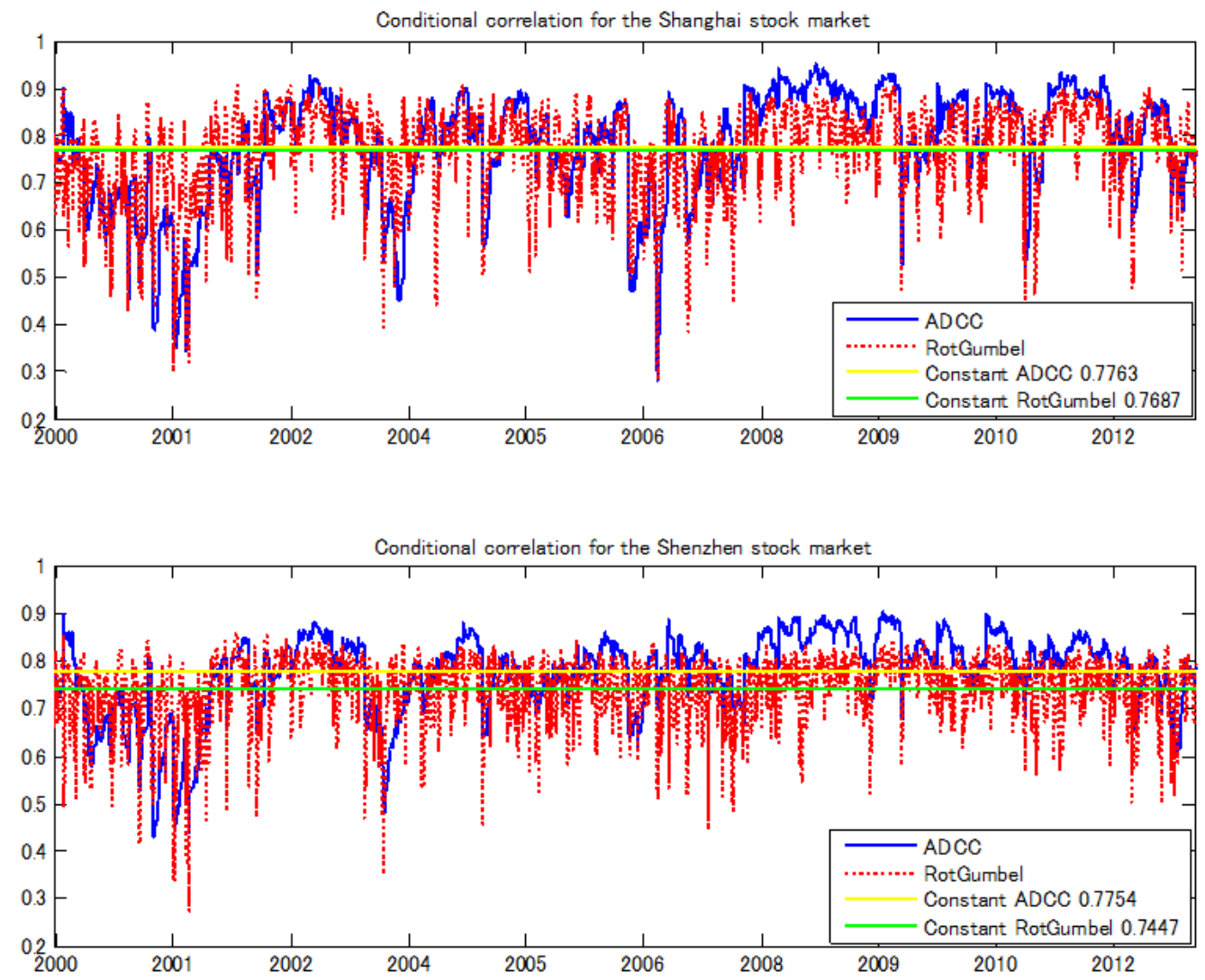

Figure 2. Dynamic Conditional Correlation Time-Varying Rotated-Gumbel Copula Parameter for the Shanghai SE (a) and Shenzhen SE (b)

\section{Concluding Remarks}

In this study, we employ the ADCC and time-varying conditional copula-GARCH models to investigate the segmentation of Chinese stock markets. In particular, we empirically examine the dynamic dependence structure between the daily stock returns of A and B shares of the Shanghai and Shenzhen SEs and make the following three findings. First, we find significant variability in the dependences between the A and B shares for both SEs. Regardless of which copula function we use, we consistently find that the A and B shares of the Shanghai market have not only higher levels of dependence but also greater variability of dependence than those of the Shenzhen SE, which implies that the A shares are more integrated with the B shares for the former compared with the latter. This outcome provides useful insights for domestic and international investors into how to practice active portfolio risk management in Chinese stock markets.

Second, based on the results of the CvM goodness-of-fit test, we suggest that time-varying rotated-Gumbel copula functions fit the dependence structure more appropriately than time-varying Student's t-copula functions for Chinese stock markets, demonstrating that the dynamic conditional dependence structures of both markets are asymmetric and lower conditionally tail-dependent.

Finally, we find that the conditional correlations between the A and B shares for the two markets increased tremendously around 2001, indicating that the commonality between the A and B shares was primarily based on the information advantage of the former. Since the opening up of the B shares market to Chinese citizens in 2001, common influences have become the main source of the rising dependences and correlations. In addition, we observe increasing dependence and correlations between the A and B shares of both markets since the 2007-2008 global financial crisis.

These findings have important implications for investors' asset allocation and portfolio management. Greater dependences during bear markets imply that opportunities for portfolio diversification reduce at such times. 
Understanding the distinction in the integration of the A and B shares of the Shanghai and Shenzhen SEs is thus useful for policymakers formulating strategies.

Our study also has some limitations. The first is that using only goodness-of-fit tests to compare the two time-varying copula models is insufficient. Hence, further tests should be carried out when making the model selection. Second, the time-varying copula functions provide a large degree of flexibility when modelling the dynamic interdependence between two assets. However, copula models are restricted to one timescale. Indeed, international investors differ depending on the investment horizon. Thus, further research should aim to use wavelet analysis to decompose the time series over different timescales.

\section{Acknowledgements}

We are grateful to the three referees for their helpful comments and suggestions.

\section{References}

Bailey, W. (1994). Risk and return on China's new stock markets: Some preliminary evidence. Pacific Basin Finance Journal, 2, 243-260. http://dx.doi.org/10.1016/0927-538X(94)90019-1

Bollerslev, T. (1986). Generalized autoregressive conditional heteroskedasticity. Journal of Econometrics, 31(3), 307327. http://citeseerx.ist.psu.edu/viewdoc/summary?doi=10.1.1.161.7380

Cappiello, L., Engle, R. F., \& Sheppard, K. (2006). Asymmetric dynamics in the correlations of global equity and bond returns, Journal of Financial Econometrics, 4(4), 537-572. http://dx.doi.org/ 10.1093/jjfinec/nbl005

Chien, M. S., Lee, C. C., Hu, T. C., \& Hu, H. T. (2015). Dynamic Asian stock market convergence: Evidence from dynamic cointegration analysis among China and ASEAN-5. Economic Modelling, 51, 84-98. http://dx.doi.org/10.1016/j.econmod.2015.06.024

Dias, A., \& Embrechts, P. (2004). Dynamic copula models for multi-variate high-frequency data in finance. Discussion paper.

Engle, R. F. (2002). Dynamic conditional correlation: A simple class of multivariate generalized autoregressive-conditional heteroskedasticity models. Journal of Business and Economic Statistics, 20(3), 339-350. http://dx.doi.org/10.1198/073500102288618487

Glosten, L. R., Jagannathan, R., \& Runkle, D. E. (1993). On the relation between the expected values and the volatility of the nominal excess return on stocks. Journal of Finance, 48(5), 1779-1801. http://dx.doi.org/ 10.1111/j.1540-6261.1993.tb05128

Gumbel, E. (1960). Distributions des valeurs extrêmes en plusiers dimensions. Publications de l'Institut de Statistique de l'Université de Paris, 9, 171-173.

Hansen, B. E. (1994). Autoregressive conditional density estimation. International Economic Review, 35(3), 705-730. http://dx.doi.org/ 10.2307/2527081

Hu, J. (2010). Dependence structures in Chinese and U.S. financial markets: A time-varying conditional copula approach. Applied Financial Economics, 20(7), 561-583. http://dx.doi.org/ 10.1080/09603100903459865

Jin, X. (2015). Volatility transmission and volatility impulse response functions among the Greater China stock markets. Journal of Asian Economics, 39, 43-58. http://dx.doi.org/ 10.1016/j.asieco.2015.05.004

Li, H. (2013). Integration versus segmentation in China's stock market: An analysis of time-varying beta risks. Journal of International Financial Markets, Institutions and Money, 25(13), 88-105. http://dx.doi.org/10.1016/j.intfin.2013.01

Luo, Y., Ren, J., \& Wang, Y. (2015). Misvaluation comovement, market efficiency and the cross-section of stock returns: Evidence from China. Economic Systems, 39(3), 390-412. http://dx.doi.org/ 10.1016/j.ecosys.2015.01.001

Nelsen, R. B. (1999). An introduction to copulas. New York: Springer Verlag.

Patton, A. J. (2006a). Estimation of multivariate models for time series of possibly different lengths. Journal of Applied Econometrics, 21, 147-173. http://dx.doi.org/ 10.1002/jae.865View/save citation

Patton, A. J. (2006b). Modeling asymmetric exchange rate dependence. International Economic Review, 47(2), 527-556. http://dx.doi.org/ 10.1111/j.1468-2354.2006.00387.xView/save citation

Sklar, A. (1959). Fonctions de repartition à n dimensions et leurs marges. Publications de l'Institut de Statistique de l'Universite de Paris, 8, 229-231. http://dx.doi.org/ 10.12691/ijefm-3-2-3

Sun, Q., \& Tong, W. H. S. (2000). The effect of market segmentation on stock prices: The China syndrome. Journal of Banking \& Finance, 24(12), 1875-1902. http://dx.doi.org/10.1016/S0378-4266(99)00121-1 
Tamakoshi, G., \& Hamori, S. (2013). An asymmetric DCC analysis of correlations among bank CDS indices. Applied Financial Economics, 23(6), 475-481. http://dx.doi.org/10.1080/09603107.2012.727973

Taylor, S. J. (1986). Modelling financial time series. Chichester: John Wiley and Sons Ltd.

Toyoshima, Y., \& Hamori, S. (2013). Asymmetric dynamics in stock market correlations: Evidence from Japan and Singapore. Journal of Asian Economics, 24, 117-123. http://dx.doi.org/ 10.1016/j.asieco.2012.08.001

Toyoshima, Y., Nakajima, T., \& Hamori, S. (2013). Crude oil hedging strategy: New evidence from the data of the financial crisis. Applied Financial Economics, 23(12), 1033-1041. http://dx.doi.org/10.1080/09603107.2013.788779

Toyoshima, Y., Tamakoshi, G., \& Hamori, S. (2012). Asymmetric dynamics in correlations of treasury and swap markets: Evidence from the US market. Journal of International Financial Markets, Institutions and Money, 22(2), 381-394. http://dx.doi.org/10.1016/j.econmod.2015.08.017

Wang, K., Chen, Y. H., \& Huang, S. W. (2011). The dynamic dependence between the Chinese market and other international stock markets: A time-varying copula approach. International Review of Economics \& Finance, 20(4), 654-664. http://dx.doi.org/10.1016/j.iref.2010.12.003

Xu, C. K. (2000). The microstructure of the Chinese stock market. China Economic Review, 11(1), $79-97$. http://dx.doi.org/ 10.1016/S1043-951X(99)00013-9

Zakoian, J. M. (1994). Threshold heteroskedastic models. Journal of Economic Dynamics and Control, 18(5), 931-955. http://dx.doi.org/ 10.1016/0165-1889(94)90039-6

Zhu, H., Li, Z., You, W., \& Zeng, Z. (2015). Revisiting the asymmetric dynamic dependence of stock returns: Evidence from a quantile autoregression model. International Review of Financial Analysis, 40, 142-153. http://dx.doi.org/10.1016/j.irfa.2015.05.013

\section{Copyrights}

Copyright for this article is retained by the author(s), with first publication rights granted to the journal.

This is an open-access article distributed under the terms and conditions of the Creative Commons Attribution license which permits unrestricted use, distribution, and reproduction in any medium, provided the original work is properly cited. 